\title{
IMPACT OF GENETICS ON NEOADJUVANT THERAPY WITH COMPLETE PATHOLOGICAL RESPONSE IN METASTATIC COLORECTAL CANCER: CASE REPORT AND REVIEW OF THE LITERATURE
}

Bulajic $\mathrm{P}^{1}$, Bidzic $\mathrm{N}^{1, *}$, Djordjevic $\mathrm{V}^{1}$, Ceranic $\mathrm{M}^{1,2}$, Basaric $\mathrm{D}^{1,2}$, Pesic $\mathrm{V}^{3}$, Djordjevic-Pesic $\mathrm{J}^{4}$

*Corresponding Author: Nemanja Bidzic, M.D., Clinic for Digestive Surgery, Clinical Center of Serbia, Koste Todorovica 6, Belgrade 11000, Serbia. Tel. +381-11-306-5957. Fax: +381-11-306-5967.

E-mail: nemanja bidzic@yahoo.com

\begin{abstract}
Treatment of colorectal metastatic cancer is still challenging, despite recent improvements in chemotherapy. A genetic cancer profile, such as the $K R A S$ (Kirsten rat sarcoma) gene status, plays a key role in individualized tailored therapy. Molecular targeted therapy added to neoadjuvant chemotherapy can achieve a better pathological response and prolong survival. Pathological complete response of colorectal cancer stage IV is rare. A 47-year-old female patient presented with rectal adenocarcinoma and three liver metastases (cT3d/4, N2, M1). After seven cycles of Bevacizumab and CAPOX in neoadjuvant setting, we noted more than $70.0 \%$ regression of metastases and complete regression of the primary tumor. We performed low anterior resection of rectum and synchronous subsegmental resection of $\mathrm{S} 3$, because the other two lesions were not detectable. Pathology revealed complete response of the primary and also secondary tumors. After 8 months, diagnostic tests did not show any sign of recurrence and the remaining liver lesions disappeared. Colorectal cancer is a heterogeneous disease and it is necessary to identify patients who are at-risk of recurrence and suitable for neoadjuvant therapy. Genetic biomarkers play an important role in metastatic colorectal cancer treatment. Because of the mutated $K R A S$ gene, Bevacizumab was added to cytotoxic therapy achieving a complete pathological response
\end{abstract}

\footnotetext{
${ }^{1}$ Clinic for Digestive Surgery, The First Surgical Clinic, Clinical Center of Serbia, Belgrade, Serbia

${ }^{2}$ Medical Faculty, University of Belgrade, Belgrade, Serbia

${ }^{3}$ University of Belgrade, Faculty of Agriculture, Department of Genetics, Belgrade, Serbia

${ }^{4}$ Medical School "Dr. Milenko Hadzic," Nis, Serbia
}

of primary tumor and metastasis. This case is unique because all reported cases with similar results, described staged surgery and one of reverse staged surgery, but with similar results. This neoadjuvant therapy has extraordinary results for colorectal cancer stage IV and can help diseasefree and long-term survival.

Keywords: Bevacizumab; KRAS (Kirsten rat sarcoma) gene mutation; Metastatic colorectal cancer; Pathological complete response; Vanishing metastases.

\section{INTRODUCTION}

Colorectal cancer is a very common malignancy and a significant health problem [1]. At the time of diagnosis, almost $25.0 \%$ of patients have synchronous metastases, and up to $50.0 \%$ will experience metastases. The liver is certainly the most common site for metastasis to occur, and about four-fifths of the patients have isolated metastatic disease in the liver [2]. However, a minority of these are initially eligible for surgery. Despite the modern improvement of cancer therapy [3], treatment of metastatic colorectal cancer is still challenging, especially in synchronous presentation. Long-term survival is poorer than in patients with metachronous metastases [4]. In the past decade, some neoadjuvant therapies have improved treatment of stage IV colorectal cancer [5]. Besides the radio and chemotherapy, newly designed biological agents have shown great advance in the management of colorectal disease, particularly in the neoadjuvant setting [6-8].

Because of the different pathways in tumor genesis and tremendous heterogeneity [9], it is necessary to stratify every single neoplastic process. Molecular targeted therapy is based on the specific genetics of every neoplastic process which implies individualized treatment according to detected genetic mutations. The role of some biomarkers, 
such as the $K R A S$ (Kirsten rat sarcoma) gene mutation, is evaluated in determining molecular targeted therapy [6].

Neoadjuvant chemotherapy response is very important for disease-free and overall survival, and in the era of effective chemotherapy, various criteria for tumor response are established [10]. A complete pathologic response of both primary and secondary tumors, is extremely rare [11]. We present a case of individualized management of metastatic colorectal cancer with a complete pathological response of both primary and secondary tumors.

Case Presentation. A 47-year-old female with a few months history of abdominal pain and bloody stools was admitted to our clinic in April 2017. Colonoscopy revealed a large circumferential tumor, $7 \mathrm{~cm}$ from the anal verge, and pathological examination of biopsied material diagnosed adenocarcinoma [Figure 1(a)]. Carcinoembryonic antigen (CEA) was $16.3 \mu / \mathrm{L}$ and carbohydrate antigen 19-9 (CA19-9) was in the normal range.

Computerized tomography (CT) of the abdomen and pelvis showed three metastatic lesions in the liver: in segment three (S3) $42 \times 33 \mathrm{~mm}$ [Figure 1(b)], in S7 two lesions, one superficial $31 \times 19 \mathrm{~mm}$ [Figure 1(c)], and the other $33 \times 33 \mathrm{~mm}$ [Figure $1(\mathrm{~d})$ ], inseparable from the right hepatic vein; the rectal tumor was in the middle and proximal part of the rectum and distal part of the sigmoid colon, $10 \mathrm{~cm}$ in length, $7 \mathrm{~cm}$ from the anal verge, calculated on CT scans [Figure 1(e) and (f)]. Clinical tumor-nodemetastasis (TNM) classification was $\mathrm{T} 3 \mathrm{~d} / 4, \mathrm{~N} 2, \mathrm{M} 1$, and one lesion of $3 \mathrm{~cm}$ was also detected in the spleen but with an unclear diagnosis. The multidisciplinary team decided to start treatment with preoperative chemotherapy. Due to the prediction of bowel obstruction, we first performed a bipolar colostomy.

The mutation was detected by genetic analysis of biopsied tumor tissue that was based on real-time polymerase chain reaction (PCR). The detected mutation was on codon $13(\mathrm{G} 13 \mathrm{X})$ of the $K R A S$ gene, but we were not able to elucidate the exact mutation type because of the method limitations. Thus, we excluded the possibility of Cetuximab according to our national guidelines. In this situation, we decided to use combined regimen of Oxaliplatin and Capecitabine with the addition of Bevacizumab, which is the only remaining biological agent for colorectal liver metastases at our oncological practice. We did not have the possibility of performing vascular endothelial growth factor (VEGF) expression on the tumor sample, so we were unable to determine tumor tissue sensitivity to Bevacizumab. However, it is widely accepted to include this medicine in the first line therapy. After four cycles of neoadjuvant therapy, we confirmed partial regression on CT scans, according to response evaluation criteria in solid tumors (RECIST), and continued with three more cycles because the patient was in good general condition and therapy was efficient.

In November 2017, CT of the thorax, abdomen, and pelvis was done and revealed total regression of the primary rectal tumor [Figure 1 (g) and (h)], and further regression of metastatic lesions (lesion in S3 was $13 \times 12$ $\mathrm{mm}$ ) [Figure 1(i)], superficial lesion in $\mathrm{S7}$ was $11 \times 8 \mathrm{~mm}$ [Figure 1(j)], and in S7 lesion near to the right hepatic vein was $8 \times 6 \mathrm{~mm}$ [Figure 1(k)]. Tumor markers reached normal values, and the splenic lesion was stable in size.

The patient was prepared for the operation, and in January 2018 , we performed a low anterior resection of the rectum with total mesorectal excision, colorectal anastomosis, and synchronous liver resection. During the operation, we did not find any signs of dissemination in the abdomen. The rectal tumor was presented as a few centimeter scar. In the liver, we found only the lesion in S3, and another two lesions were not detectable either by palpation or by intraoperative ultrasound. Thus, we performed only a subsegmental liver resection of $\mathrm{S} 3$, and because the CT scan detected the splenic lesion, we also performed a splenectomy.

The patient recovered from the operation without any complication. Pathological examination showed the complete response of both primary and secondary liver tumors without any positive lymph node (yTNM8: T0, N0 $(0 / 31), M 0)$. Microscopic findings described only fibrosis and mucin in rectal tumor residue [Figure 1(1)] and multiple necrotic nodules surrounded by fibro-vascular septa in the liver specimen [Figure $1(\mathrm{~m})$ ]. The splenic tumor was characterized to be a benign sclerotic vascular tumor.

In the follow-up period after 4 and 8 months we did not find any sign of malignancy. Four months after the operation, tumor markers were normal, and we found S3 without recurrence on the CT scan [Figure 1(n)] and two lesions in S7 that are described as fibrotic tissue [Figure 1(o) and (p)]. After 8 months, the CT scan did not show any sign of focal lesions in the liver and tumor markers were still in the normal range. Then we performed positronemission tomography (PET) and confirmed that the liver was free of malignancy and pelvis free of recurrent tumors. The patient is still free of recurrent disease.

\section{DISCUSSION}

Today standard therapy for metastatic colorectal cancer certainly comprise both surgery and chemotherapy. Outcomes of surgery alone include death in almost $30.0 \%$ of patients during the first 2 years. Chemotherapy has not yet achieved a long-term cure [2,4], hence, surgery is still necessary for cure. With combined treatment, more than $50.0 \%$ of the patients can reach a 5-year survival and up 


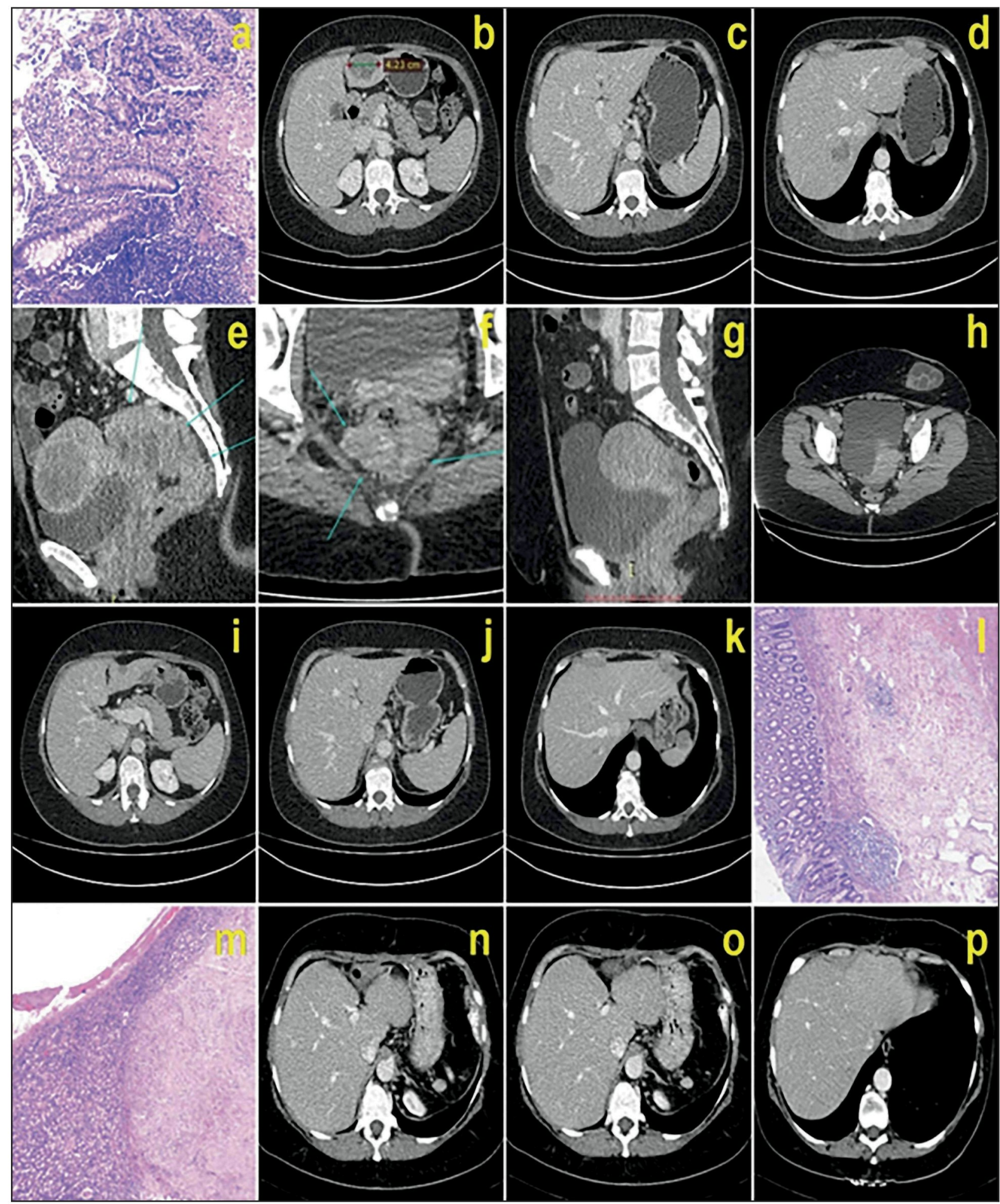

Figure 1. (a): Preoperative histopathology-adenocarcinoma; (b), (c), (d), (e), (f): preoperative CT of primary and secondary tumors; (g), (h), (i), (j), (k): CT after neoadjuvant therapy; (l), (m): complete pathological response of primary rectal tumor and secondary liver metastases in S3, respectively; (n), (o), (p): CT shows vanishing liver metastases 4 months after the operation.

to $25.0 \% 10$-year survival [12]. Even then, the recurrence rate is high, and about $75.0 \%$ of all recurrences are seen in the first 2 years [2].
Chemotherapy can prolong recurrence-free survival $[4,13]$, increase resectability of liver metastases [14] and has an impact on overall survival [15]. Different regimens 
of cytotoxic agents are in use (FOLFOX, CAPOX, FOLFIRI, and FOLFOXIRI). New biological therapy added to cytotoxic regimens may enhance therapeutic efficiency, especially in the neoadjuvant setting $[16,17]$. Chemotherapy, with or without targeted therapy, is recommended for synchronous colorectal liver metastases because they have a less favorable cancer biology $[13,16]$ and expected survival than metachronous, particularly in late metachronous metastases [2].

It is of great importance to identify patients at-risk of recurrence and the patients who will receive a benefit from neoadjuvant therapy. Earlier clinical and pathological risk scores are used to estimate prognosis after surgery and to evaluate eligibility for surgery [4]. In the era of modern systemic chemotherapy regimens, these risk scores have low efficiency in clinical decision-making $[18,19]$.

Colorectal cancer is a heterogeneous disease with several possible pathways responsible for carcinogenesis and a lot of genetic mutations. Different genetic and chromosomal alterations are described: microsatellite instability, loss of heterozygosity of chromosome 18, mutations in p53, RAS and RAF mutations (9). Thus, various tumor biology is possible and subsequently, different responses to neoadjuvant therapy. Molecular biomarkers are now being incorporated in risk stratification and treatment decision due to developing a personalized or individualized treatment [20]. Testing of cancer genetic profile has a leading role in the new concept of personalized medicine, and some of these are widely accepted and frequently used in molecular targeted therapy [21].

Evaluated molecular targeted therapy for metastatic colorectal cancer includes Bevacizumab and Cetuximab. Bevacizumab is a monoclonal antibody inhibitor of VEGF, which is the product of the same-named gene and has a crucial role in angiogenesis and tumor growth $[7,8]$. Cetux-imab is a monoclonal antibody inhibitor of epidermal growth factor receptor (EGFR) which is very effective in the therapy of $K R A S$ wild-type cancers but with no effect on $K R A S$ mutations $[6,21]$, except in the specific mutation on codon 13 (G13D), which is probably associated with some response to Cetuximab [22]. In the present case, we detected a mutation on codon 13 but without differentiation of the exact mutation type, and we did not know if it was a G13D mutation. Even then, the results of recent studies were not so strong to incorporate the Cetuximab for G13D, especially in a neoadjuvant setting [22]. We decided to use cytotoxic neoadjuvant therapy (CAPOX) and to add Bevacizumab as the only remaining biological agent for colorectal liver metastases, which were previously evaluated as a potent neoadjuvant regimen. There is a possibility for estimation of VEGF expression in the tumor sample, but in our oncological practice it is not routinely used for colorectal cancer as the majority of patients have good therapeutic results $[8,16,17]$. After seven cycles of CAPOX and Bevacizumab, we achieved an extraordinary response: more than $70.0 \%$ regression of metastatic lesions and complete regression of the primary tumor, according to RECIST. Carcinoembryonic antigen and CA19-9 were normal. Postoperative histopathology revealed complete primary and secondary tumor response without any evidence of malignancy [Figure 1(l) and $1(\mathrm{~m})$ ].

The complete pathological response has been reported, but most of the authors have presented cases with staged surgery for synchronous metastatic disease and input neo-adjuvant treatment after resection of the primary tumor and before resection of secondary tumors [23-25]. In one case, it was even a reverse or liver-first approach but also achieving complete response in primary and metastatic tumors [26].

The primary tumor was locally advanced (cT3d/4,N2) and surgery as a first-line treatment would not be a proper decision because of the risk of local recurrence. On the other hand, pelvic radiotherapy has a complete response in 10.0 to $20.0 \%$ cases but has many side effects [27]. In this case, we had to treat both primary and metastatic tumors and achieving a positive effect on neoadjuvant treatment we achieved a chance for a synchronous resection.

Vanishing metastases and impossibility to detect liver lesions intraoperatively after neoadjuvant treatment have been described. The reason is the same echogenicity of the lesion and normal liver parenchyma [28]. Concordance between imaging and pathological examination particularly in bevacizumab-containing neoadjuvant therapy is poor and so visible lesions on imaging can be without malignant cells on histopathology [29]. Natural history of undetectable lesions after neoadjuvant chemotherapy in case of complete pathological response of the other lesions has not yet been elucidated. We performed CT scans, tumor markers and PET after 8 months and all pointed to recurrence-free status. Complete disappearance of primary and secondary lesions makes this case unique.

\section{CONCLUSIONS}

There are literature data on similar efficiency of combined cytotoxic and biological therapy, especially in the neoadjuvant setting, as well as on its impact on long-term survival. Genetic mutations as biomarkers have a crucial role in the management of colorectal cancer stage IV, with $K R A S$ mutation status being significant in the choice of neoadjuvant molecular targeted therapy. Our study has shown that Bevacizumab-containing neoadjuvant treatment can have rather promising results for both metastatic and primary colorectal cancer. 
Pathological complete response is associated with better overall survival and should be evaluated in future research. We conclude that modern cancer therapy must be individualized, multimodal and based on a genetic cancer profile.

Declaration of Interest. The authors report no conflicts of interest. The authors alone are responsible for the content and writing of this article.

\section{REFERENCES}

1. Ferlay J, Soerjomataram I, Dikshit R, Eser S, Mathers $\mathrm{C}$, Rebelo $\mathrm{M}$, et al. Cancer incidence and mortality worldwide: Sources, methods and major patterns in GLOBOCAN 2012. Int J Cancer. 2015; 136(5): E359E386.

2. Tomlinson JS, Jarnagin WR, DeMatteo RP, Fong $Y$, Kornprat $\mathrm{P}$, Gonen $\mathrm{M}$, et al. Actual 10-year survival after resection of colorectal liver metastases defines cure. J Clin Oncol. 2007; 25(29): 4575-4580.

3. André T, Boni C, Mounedji-Boudiaf L, Navarro M, Tabernero J, Hickish T, et al. Oxaliplatin, fluorouracil, and leucovorin as adjuvant treatment for colon cancer. N Engl J Med. 2004; 350(23): 2343-2351.

4. Fong Y, Fortner J, Sun RL, Brennan MF, Blumgart LH. Clinical score for predicting recurrence after hepatic resection for metastatic colorectal cancer: Analysis of 1001 consecutive cases. Ann Surg. 1999; 230(3): 309-318.

5. Nordlinger B, Sorbye H, Glimelius B, Poston GJ, Schlag PM, Rougier $\mathrm{P}$, et al. Perioperative chemotherapy with FOLFOX4 and surgery versus surgery alone for resectable liver metastases from colorectal cancer (EORTC 40983): A randomised, controlled trial. Lancet. 2008; 37(9617): 1007-11016.

6. Bokemeyer C, Bondarenko I, Hartmann JT, de Braud F, Schuch G, Zubel A, et al. Efficacy according to biomarker status of cetuximab plus FOLFOX-4 as first-line treatment for metastatic colorectal cancer: The OPUS study. Ann Oncol. 2011; 22(7): 1535 1546.

7. Saltz LB, Clarke S, Díaz-Rubio E, Scheithaure W, Figer A, Wong $\mathrm{R}$, et al. Bevacizumab in combination with oxaliplatin-based chemotherapy as first-line therapy in metastatic colorectal cancer: A randomized phase III study. J Clin Oncol. 2008; 26(12): 20132019.

8. Gruenberger B, Tamandl D, Schueller J, Scheithauer W, Zielinski C, Herbst F, et al. Bevacizumab, capecit- abine, and oxaliplatin as neoadjuvant therapy for patients with potentially curable metastatic colorectal cancer. J Clin Oncol. 2008; 26(11):1830-1835.

9. Manne U, Jadhav T, Putcha BK, Samuel T, Soni S, Shanmugam C, et al. Molecular biomarkers of colorectal cancer and cancer xisparities: Current status and perspective. Curr Colorectal Cancer Rep. 2016; 12(6): 332-344.

10. Rubbia-Brandt L, Giostra E, Brezault C, Roth AD, Andres A, Audard V, et al. Importance of histological tumor response assessment in predicting the outcome in patients with colorectal liver metastases treated with neo-adjuvant chemotherapy followed by liver surgery. Ann Oncol. 2007; 18(2): 299-304.

11. Adam R, Wicherts DA, de Haas RJ, Aloia T, Levi F, Paule $\mathrm{B}$, et al. Complete pathologic response after preoperative chemotherapy; myth or reality? J Clin Oncol. 2008, 26(10): 1635-1641.

12. Nordlinger B, Sorbye H, Glimelius B, Poston GJ, Schlag PM, Rougier P, et al. Perioperative FOLFOX4 chemotherapy and surgery versus surgery alone for resectable liver metastases from colorectal cancer (EORTC 40983): Long-term results of a randomised, controlled, phase 3 trial. Lancet Oncol. 2013; 14(12): 1208-1215.

13. Chua TC, Saxena A, Liauw W, Kokandi A, Morris DL. Systematic review of randomized and nonrandomized trials of the clinical response and outcomes of neoadjuvant systemic chemotherapy for resectable colorectal liver metastases. Ann Surg Oncol. 2010; 17(2): 492-501.

14. Adam R, Avisar E, Ariche A, Giachetti S, Azoulay $\mathrm{D}$, Castaing $\mathrm{D}$, et al. Five-year survival following hepatic resection after neoadjuvant therapy for nonresectable colorectal [liver] metastases. Ann Surg Oncol. 2001; 8(4): 347-353.

15. Small RM, Lubezky N, Shmueli E, Figer A, Aderka $\mathrm{D}$, Nakache $\mathrm{R}$, et al. Response to chemotherapy predicts survival following resection of hepatic colorectal metastases in patients treated with neoadjuvant therapy. J Surg Oncol. 2009; 99(2): 93-98.

16. Uetake $H$, Yasuno $M$, Ishiguro $M$, Kameoka S, Shimada Y, Takahashi $\mathrm{K}$, et al. A multicenter phase II trial of mFOLFOX6 plus bevacizumab to treat liver-only metastases of colorectal cancer that are unsuitable for upfront resection (TRICC0808). Ann Surg Oncol. 2015; 22(3): 908-915.

17. Klinger M, Tamandl D, Eipeldauer S, Hacker S, Herberger B, Kaczirek K, et al. Bevacizumab improves pathological response of colorectal cancer liver me- 
tastases treated with XELOX/FOLFOX. Ann Surg Oncol. 2010; 17(8): 2059-2065.

18. Ayez N, Lalmahomed ZS, van der Pool AE, Vergouwe $\mathrm{Y}$, van Montfort $\mathrm{K}$, de Jonge $\mathrm{J}$, et al. Is the clinical risk score for patients with colorectal liver metastases still useable in the era of effective neoadjuvant chemotherapy? Ann Surg Oncol. 2011; 18(10): 2757-2763.

19. Schreckenbach T, Malkomes P, Bechstein WO, Woeste G, Schnitzbauer AA, Ulrich F. The clinical relevance of the Fong and the Nordlinger scores in the era of effective neoadjuvant chemotherapy for colorectal liver metastasis. Surg Today. 2015; 45(12): 1527-1534.

20. Balachandran VP, Arora A, Gonen $M$, Ito $H$, Turcotte $\mathrm{S}$, Shia $\mathrm{J}$, et al. A validated prognostic multigene expression assay for overall survival in resected colorectal cancer liver metastases. Clin Cancer Res. 2016; 22(10): 2575-2582.

21. Linardou H, Dahabreh IJ, Kanaloupiti D, Siannis F, Bafaloukos D, Kosmidis $\mathrm{P}$, et al. Assessment of somatic k-RAS mutations as a mechanism associated with resistance to EGFR-targeted agents: a systematic review and meta-analysis of studies in advanced non-small-cell lung cancer and metastatic colorectal cancer. Lancet Oncol. 2008; 9(10): 962-972.

22. Schirripa M, Loupakis F, Lonardi S, Cremolini C, Bergamo F, Zagonel V, et al. Phase II study of singleagent cetuximab in KRAS G13D mutant metastatic colorectal cancer. Ann Oncol. 2015; 26(12): 25032503.

23. Yokota T, Sano T, Shimizu Y, Takahari D, Senda Y, Shimura M, et al. Pathological complete response of colorectal liver metastases following chemotherapy with S-1 and oxaliplatin (SOX) in combination with bevacizumab: A case report. Oncol Lett. 2011; 2(2): 201-205.
24. Arata $R$, Itamoto $T$, Ikeda $S$, Nakahara H, Oshita A, Shinozaki $\mathrm{K}$, et al. Pathological complete response after neoadjuvant chemotherapy for rectal cancer with synchronous multiple liver metastases: a report of an unusual case. Surg Case Rep. 2016; 2(1): 106.

25. Malavasi N, Ponti G, Depenni R, Bertolini F, Zironi $\mathrm{S}$, Luppi $\mathrm{G}$, et al. Complete pathological response in a patient with multiple liver metastases from colon cancer treated with Folfox- 6 chemotherapy plus bevacizumab: a case report. J Hematol Oncol. 2009; 2: 35 .

26. Naiken SP, Toso C, Rubbia-Brandt L, Thomopoulos $\mathrm{T}$, Roth A, Mentha $\mathrm{G}$, et al. Complete path-ological response (ypTONOM0) after preoperative chemotherapy alone for stage IV rectal cancer. BMC Surg. 2014; $14: 4$.

27. Bujko K, Nowacki MP, Nasierowska-Guttmejer A, Michalski W, Bebenek M, Kryj M. Long-term results of a randomised trial comparing preoperative shortcourse radiotherapy with preoperative conventionally fractionated chemoradiation for rectal cancer. $\mathrm{Br} \mathrm{J}$ Surg. 2006; 93(10): 1215-1223.

28. Van Vledder MG, Pawlik TM, Munireddy S, Hamper U, De Jong MC, Choti MA. Factors determining the sensitivity of intraoperative ultrasonography in detecting colorectal liver metastases in the modern era. Ann Surg Oncol. 2010; 17(10):2756-2763.

29. Chun YS, Vauthey JN, Boonsirikamchai P, Maru DM, Kopetz S, Palavecino M, et al. Association of computed tomography morphologic criteria with pathologic response and survival in patients treated with bevacizumab for colorectal liver metastases. JAMA. 2009; 302(21): 2338-2344. 